\title{
ESTRUTURA FITOSSOCIOLÓGICA EM ÁREA NATURAL E ANTROPIZADA DE UMA VEREDA EM UBERLÂNDIA, MG
}

\author{
Antônio José Maia Guimarães ${ }^{1}$ \\ Glein Monteiro de Araújo ${ }^{2}$ \\ Gilberto Fernandes Corrêa ${ }^{3}$
}

Recebido em 06/01/2001. Aceito em 22/02/2002.

\begin{abstract}
RESUMO - (Estrutura fitossociológica em área natural e antropizada de uma vereda em Uberlândia, MG). O objetivo do estudo foi de comparar a área natural e antropizada de uma vereda quanto à estrutura fitossociológica e às características do solo. O trabalho foi realizado em uma vereda no município de Uberlândia, MG (19¹1'40" S e 48²4'31” W). Para as análises químicas foram coletados solos em três profundidades: 0-1 cm, 1-16 cm e 16-32 cm. No levantamento fitossociológico utilizou-se o método transecto de linha. Duas classes de solos foram encontradas: Gleissolo Háplico e Gleissolo Melânico, que tiveram, em média, baixos valores de pH e bases disponíveis. Os maiores percentuais de matéria orgânica e umidade ocorreram no fundo da vereda. Foram amostradas 101 espécies e 29 famílias vegetais. A riqueza de espécies foi menor na vertente preservada. Poaceae, Cyperaceae e Asteraceae foram as famílias que tiveram o maior número de espécies, 33, 13 e 10, respectivamente. Schizachyrium tenerum, Echinolaena inflexa, Loudetia flammida e Erianthus asper tiveram maior frequência no ambiente preservado e Schizachyrium tenerum, Hypogynium virgatum e Ageratum fastigiatum no antropizado.
\end{abstract}

Palavras-chave - Fitossociologia, solos, vereda

\begin{abstract}
Phytosociological structure of natural and disturbed palm swampy vegetation near Uberlândia, MG). The study aimed to compare the natural and disturbed areas of a palm swamp vegetation according to its soil characteristics and phytosociological structure. The study was done at a site near Uberlândia, MG (19¹1'40" S and 48²4’31” W). Chemical analysis was carried out at threes depths: 0-1 cm, 1-16 cm and 16-32 cm. The phytosociological survey was done by the line intercept method. Two classes of soil were found: Relatively Humic Glei and Humic Glei, which had low values of $\mathrm{pH}$ and available bases. The highest percentage of organic matter
\end{abstract}

1 Bolsista CAPES. MSc. em Ecologia e Conservação dos Recursos Naturais pela Universidade Federal de Uberlândia, MG. veredaambiental@uol.com.br

2 Instituto de Biologia, Universidade Federal de Uberlândia, Caixa Postal 593, 38400-902, Uberlândia, MG, Brasil. glein@ufu.br

3 Instituto de Agronomia, Universidade Federal de Uberlândia, Caixa Postal 593, 38400 - 902, Uberlândia, MG, Brasil. gfcorrea@ufu.br 
occurred in the lowest depth of the palm swamp area. Some 101 species and 29 families were sampled. The preserved slope had a lower species richness than the disturbed area. Poaceae, Cyperaceae and Asteraceae were the families with the highest number of species, 33, 13 and 10, respectively. Schizachyrium tenerum, Echinolaena inflexa, Loudetia flammida and Erianthus asper were more frequent in the preserved area and Schizachyrium tenerum, Hypogynium virgatum and Ageratum fastigiatum in the disturbed area.

Key words - Phytosociological, soils, palm swamp

\section{Introdução}

O bioma cerrado constitui-se em seu conjunto uma região fitogeográfica desuniforme, apresentando em sua paisagem inclusões de formações florestais, em meio às formações savânicas e campestres (Ferreira 1980). Dentre os diversos tipos fisionômicos de vegetação nessa região, existem as veredas que ocorrem em geral, em áreas de nascentes, com elevado nível de umidade no solo. Estas representam um ecossistema de grande relevância na região do cerrado (Carvalho 1991).

As veredas são comunidades hidrófilas formadas por dois tipos de vegetação: uma herbáceo-graminosa que ocupa a maior parte de sua área, e outra arbórea-arbustiva com predominância dos buritis (Carvalho 1991). O buriti (Mauritia flexuosa) é uma espécie de palmeira, de porte arbóreo, que carateriza as veredas da região dos cerrados do Brasil Central (Magalhães 1956). Segundo Ramirez \& Brito (1990), os buritizais definem as áreas tropicais brejosas, já que diferem facilmente da vegetação de cerrado que os cerca. Em fases mais evoluídas, podem ser encontradas na vereda, junto aos buritis, ilhas de vegetação lenhosa. Quando os vales tornam-se mais encaixados e o lençol freático é rebaixado, as veredas dão lugar às matas ciliares (Melo 1992).

A distribuição geográfica das veredas, no domínio dos cerrados, abrange as bacias dos rios Paranaíba, São Francisco e Grande, compreendendo as regiões do Triângulo Mineiro, Alto Paranaíba, Alto São Francisco e Paracatu (Carvalho 1991), mas também podem ser abundantes em todas as chapadas do Brasil Central
(Lima \& Queiróz Neto 1996). No Triângulo Mineiro, as veredas ocorrem em diferentes superfícies geomorfológicas (Lima 1996; Lima \& Queiroz Neto 1996; Ramos 2000). Em um levantamento realizado para diagnosticar a ocupação e uso de solo, Lima et al. (1989) revelaram que em Uberlândia os campos hidromórficos e veredas ocorrem em áreas de alta concentração de umidade e matéria orgânica no solo, distribuindo-se por $6,68 \%$ do município.

Estudos florísticos abrangendo vários tipos fisionômicos de vegetação de cerrado, incluindo parcialmente áreas de vereda, foram realizados por Pereira et al. (1990), Silva Júnior \& Felfili (1996) e Mendonça et al. (1998) no Distrito Federal e Brandão \& Gavilanes (1994) no Triângulo Mineiro. A flora de ambientes similares às veredas denominados de "morichales", foi estudada por Aristeguieta (1968) na Venezuela.

Com a crescente ocupação da região do cerrado pela agricultura "moderna", as comunidades vegetais de vereda têm sofrido alterações de natureza antrópica, que em alguns casos tornam-se irreversíveis, devido principalmente à sua pequena capacidade de regeneração (Carvalho 1991).

Além da importância ecológica e hidrológica das veredas, pode-se destacar o seu valor paisagístico, e o papel social exercido pelas mesmas para pequenas comunidades de agricultores que exploram sustentavelmente a palmeira buriti, para diversas finalidades (Fonseca \& Silva 1998). Devido a importância deste ecossistema, e por tratar-se de um ambiente sensível a alterações (Boaventura 1988), as legislações Federal e Estadual o reconhece como área protegida por lei, ou seja, Área de Preservação Permanente (Brasil 1992; Minas Gerais 1988). 
O objetivo desse trabalho foi comparar a área natural e antropizada de uma vereda situada no município de Uberlândia, Minas Gerais, quanto, à estrutura fitossociológica e às características do solo.

\section{Material e métodos}

A vereda estudada localiza-se na porção sul do município de Uberlândia, MG, inserida na bacia hidrográfica do Rio Tijuco, nas coordenadas geográficas de $19^{\circ} 11^{\prime} 40^{\prime \prime} \mathrm{S}$ e $48^{\circ} 24^{\prime} 31^{\prime \prime} \mathrm{W}$. Constitui-se uma das nascentes do Córrego Santa Maria e possui uma vertente preservada localizada na Estação Ecológica do Panga (EEP) e outra antropizada, localizada em propriedade particular denominada Fazenda Tarapoto (Fig. 1). Esta propriedade rural possui como principal atividade a pecuária extensiva que se estende até a área da vereda estudada. A Estação Ecológica do Panga é uma Unidade de Conservação registrada no Ibama como Reserva Particular do Patrimônio Natural (RPPN), e possui uma área de 409,5 ha. Cerca de $9 \%$ de sua área é formada por veredas e campos úmidos (Schiavini \& Araújo 1989).

O solo da área cincunvizinha é predominantemente Latossolo Vermelho Distrófico textura média fase cerrado (Embrapa 1999). Os solos hidromórficos ocorrem no fundo dos vales (Lima et al. 1989) e nas vertentes com surgência do lençol freático (Baccaro 1994).

O clima regional, segundo a classificação de Köppen (Embrapa 1982), é do tipo Aw (megatérmico), com duas estações bem definidas: uma seca, com período de estiagem que vai de maio a agosto, e outra úmida que se estende de novembro a março. A precipitação pluviométrica média é de $1.600 \mathrm{~mm}$ anuais, com maior incidência nos meses de dezembro e janeiro (Rosa et al. 1991).

Para caracterização das propriedades químicas, amostrou-se o solo nas profundidades de
0-1 cm, 1-16 cm e 16-32 cm, mediante abertura de 12 trincheiras sendo 6 distribuídas na área preservada e 6 na área antropizada. As profundidades de amostragem limitaram-se a 32 centímetros por corresponder ao horizonte diagnóstico A (nos solos Glei) e à porção superior do horizonte H (nos solos Orgânicos) (Ramos 2000). As análises químicas foram realizadas no Laboratório de Análises de Solo, Fertilizante, Calcário e Foliar, do Instituto de Ciências Agrárias da Universidade Federal de Uberlândia, seguindo-se a metodologia de rotina (Embrapa 1979).

Para a amostragem da vegetação foram estabelecidos três transectos, de montante para jusante da vereda, tendo cada um respectivamente $85 \mathrm{~m}, 100 \mathrm{~m}$ e $115 \mathrm{~m}$ de comprimento (Fig 1). A distância aproximada entre eles foi de 36 metros do primeiro para o segundo e 55 metros entre o segundo e o terceiro. A posição dos transectos foi perpendicular à linha de drenagem, sendo que parte deles encontrou-se na vertente preservada e outra na antropizada (Fig. 1).

O perfil topográfico dos transectos foi demarcado utilizando-se uma mangueira de nível.

O levantamento fitossociológico foi realizado utilizando-se o método transecto de linha (Brower \& Zar 1984). Em cada transecto estendeu-se uma linha da borda direita à borda esquerda da vereda, totalizando 300 metros de vegetação amostrada nos dois ambientes. Os três transectos representados pelas linhas foram divididos em seções de $1 \mathrm{~m}$, o que resultou em um total de 300 seções. Para cada seção de um metro registrou-se as espécies interceptadas pela linha, o espaço ocupado pelas mesmas, bem como a altura média e o percentual de biomassa viva e morta.

As espécies amostradas foram identificadas por especialistas ou por comparação com exsicatas existentes no herbário da Universidade Federal de Uberlândia (HUFU) e da Universidade de Brasília (UB). O material com flor e/ ou fruto coletado, encontra-se depositado no Herbarium Uberlandensis (HUFU) da Univer- 


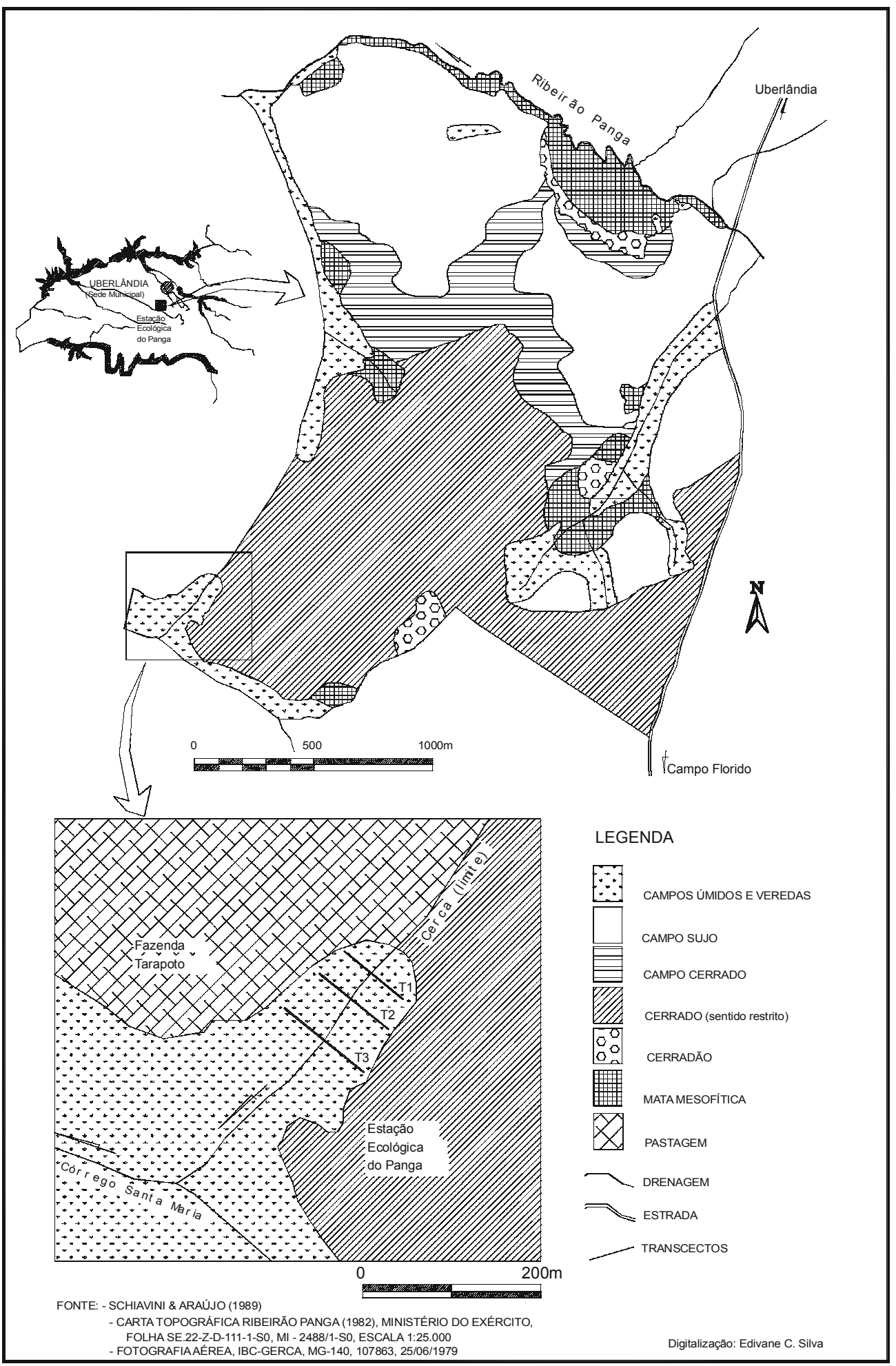

Figura 1. Mapa de localização da Estação Ecológica do Panga e da área de estudo no município de Uberlândia, MG. 
sidade Federal de Uberlândia, MG. Os parâmetros fitossociológicos para as espécies (frequiência relativa e cobertura relativa) foram calculados segundo Brower \& Zar (1984). Para os testes de similaridade florística entre as espécies amostradas, nos dois ambientes estudados, utilizou-se o Índice de Sorensen (MuellerDombois \& Ellenberg 1974).

O teste de $t$ (Zar 1984) foi utilizado para comparação de médias entre as áreas natural e antropizada para frequiência e cobertura relativa, altura média da vegetação e porcentagem de matéria viva.

\section{Resultados e discussão}

A vereda estudada apresenta um perfil topográfico com vertentes sub-retilíneas ou ligeiramente convexas, configurando um vale aberto. A declividade média das vertentes, nos transectos 1,2 e 3 , é da ordem de $4,1 \%, 7,1 \%$ e $7,2 \%$, respectivamente. Estes valores são maiores que os descritos por Lima \& Queiroz Neto (1996) em veredas localizadas na bacia do Ribeirão do Panga, os quais foram inferiores a $3 \%$. Segundo Carvalho (1991), as características geomorfológicas das veredas podem ser determinadas pelo estágio evolutivo em que as mesmas se encontram. Veredas com maior encaixe de relevo (maiores declividades) correspondem a estágios evolutivos mais avançados, com presença de espécies arbóreas ao longo da linha de drenagem, permitindo a instalação de uma futura estrutura florestal (Carvalho 1991) .

As duas classes de solo observadas (Gleissolo Háplico e Gleissolo Melânico) se diferenciam pela expressão do horizonte de superfície (A e/ou $\mathrm{H}$ ). Este último (horizonte turfoso), no entanto, não chega a alcançar expressão para caracterizar um Solo Orgânico (Organossolo), nem mesmo na porção central da vereda. Ambos os solos encontrados são, portanto, minerais hidromórficos, pertencentes à ordem dos Gleissolos. Os resultados analíticos (Tab. 1) revelam tratar-se de solos fortemente ácidos e dessaturados (Embrapa, 1999). Os valores de $\mathrm{pH}$ e de bases trocáveis são de estreita amplitude entre os ambientes (antropizado e preservado) e, portanto, insuficientes para caracterizar variações de natureza pedológica quanto às características químicas analisadas.

A distribuição destas classes de solo ao longo da vertente, segundo Ramos (2000), pode estar relacionada com a variação do nível do lençol freático, associada ao relevo. O solo Glei Pouco Húmico, cuja extensão no transecto é de aproximadamente 35 metros, corresponde à zona de borda da vereda, no limite com o cerrado,

Tabela 1. Características químicas e matéria orgânica nos solos da área antropizada (a) e preservada (p), de uma vereda no município de Uberlândia, MG.

\begin{tabular}{|c|c|c|c|c|c|c|c|c|c|c|c|c|c|c|c|}
\hline \multirow{3}{*}{ Classes de solo } & \multirow{3}{*}{$\begin{array}{l}\text { Prof. } \\
\text { (cm) }\end{array}$} & \multirow{2}{*}{\multicolumn{2}{|c|}{$\begin{array}{l}\mathrm{pH} \\
\mathrm{H}_{2} \mathrm{O}\end{array}$}} & \multicolumn{2}{|c|}{$\mathrm{P}$} & \multicolumn{2}{|c|}{$\mathrm{K}$} & \multicolumn{2}{|c|}{$\mathrm{Ca}+\mathrm{Mg}$} & \multicolumn{2}{|c|}{$\mathrm{SB}^{1}$} & \multirow{2}{*}{\multicolumn{2}{|c|}{$\begin{array}{l}\mathrm{V}^{2} \\
-\% \\
\end{array}$}} & \multirow{2}{*}{\multicolumn{2}{|c|}{$\begin{array}{c}\text { M.O. }{ }^{3} \\
-\mathrm{g} . \mathrm{Kg}^{-1} \\
\end{array}$}} \\
\hline & & & & \multicolumn{4}{|c|}{$\mathrm{mg} \mathrm{dm}^{-3}$} & \multicolumn{4}{|c|}{ cmolc. $/ \mathrm{dm}^{-3}$} & & & & \\
\hline & & $\mathrm{a}$ & $\mathrm{p}$ & $\mathrm{a}$ & $\mathrm{p}$ & $\mathrm{a}$ & $\mathrm{p}$ & $\mathrm{a}$ & $\mathrm{p}$ & $\mathrm{a}$ & $\mathrm{p}$ & $\mathrm{a}$ & $\mathrm{p}$ & $\mathrm{a}$ & $\mathrm{p}$ \\
\hline Glei Húmico $^{4}$ & $0-1$ & 5,2 & 5,1 & 3,5 & 5,3 & 130 & 103 & 0,2 & 0,2 & 0,7 & 0,6 & 6 & 5 & 30,1 & 19,1 \\
\hline “ & $1-16$ & 4,9 & 4,8 & 3,3 & 5,0 & 37 & 43 & 0,1 & 0,1 & 0,2 & 0,3 & 2 & 2 & 12,8 & 10,7 \\
\hline “ & $16-31$ & 4,7 & 4,7 & 2,1 & 2,9 & 16 & 15 & 0,0 & 0,0 & 0,2 & 0,2 & 2 & 2 & 4,5 & 4,4 \\
\hline Glei & & & & & & & & & & & & & & & \\
\hline PoucoHúmico $^{5}$ & $0-1$ & 5,1 & 4,8 & 1,5 & 2,9 & 20 & 20 & 0,1 & 0,1 & 0,2 & 0,2 & 6 & 5 & 1,9 & 1,9 \\
\hline “ & $1-16$ & 5,0 & 4,9 & 1,6 & 1,5 & 07 & 18 & 0,0 & 0,0 & 0,1 & 0,1 & 3 & 4 & 1,5 & 1,6 \\
\hline “ & $16-31$ & 5,0 & 4,8 & 0,8 & 0,9 & 03 & 05 & 0,0 & 0,0 & 0,1 & 0,1 & 3 & 6 & 0,7 & 0,8 \\
\hline
\end{tabular}

1 = soma de bases; 2 = saturação por bases; 3 = matéria orgânica; 4 = média de quatro seções de controle e $5=$ média de cinco seções de controle. 
onde ocorre uma melhor drenagem e, consequentemente, menor teor de matéria orgânica e cor menos escura.

A ausência de processos erosivos na vertente não antropizada deve-se à presença de vegetação herbáceo-graminosa mais densa e contínua, propiciando proteção mais efetiva ao solo contra a ação direta das chuvas e o escoamento superficial.

No levantamento fitossociológico realizado nos dois ambientes da vereda, foram amostradas 101 espécies distribuídas em 70 gêneros e 29 famílias, sendo 71 espécies encontradas na área preservada e 78 na área antrópica (Tab. 2). A maior riqueza observada na área antropizada, em relação à preservada, pode ser explicada pela heterogeneidade ambiental produzida por perturbações na estrutura uniforme do ambiente. Segundo Ricklefs (1996), estes fatores são responsáveis pelas variações na diversidade de espécies vegetais.

As perturbações no ambiente, no caso específico, pastejo e pisoteio bovino, seguido por erosão superficial do solo, podem ter sido responsáveis pelas diferenças na riqueza florística entre a área antropizada e preservada. Segundo Pandey \& Singh (1991) e Belsky (1992), as áreas protegidas tendem a apresentar uma menor riqueza de espécies que áreas pastejadas correspondentes, mas esta alteração está diretamente relacionada a intensidade do pastejo (Milchunas et al. 1988). Para estes autores, a riqueza aumenta em pastejo moderado e declina em pastejo intenso. Segundo os mesmos, a maior cobertura vegetal em áreas preservadas em relação às pastejadas, ocorre devido a presença de espécies dominantes.

A similaridade florística entre os dois ambientes estudados da vereda (área antropizada e área preservada) foi de 64,4\%. De 101 espécies amostradas, 48 foram comuns aos dois ambientes, 23 exclusivas do ambiente preservado e 30 exclusivas do ambiente antropizado. Dentre as espécies exclusivas do ambiente antropizado, destacaram-se Paspalum notatum, Brachiaria decumbens, Sida rhombifolia e Emilia sonchifolia. Estas espécies são consideradas, por Gavilanes \& D'angiere Filho (1991) e Pedrotti \& Guarim Neto (1998), invasoras de áreas naturais perturbadas e são também denominadas de ruderais, comprovando que a alteração da área favorece o seu estabelecimento. Dentre as espécies exclusivas do ambiente preservado destacaram-se Cuphea linarioides, Miconia theaezans e Eriosema benthamiana, também encontradas nos estudos de Brandão \& Gavilanes (1994) em áreas naturais de vereda.

As famílias com maior número de espécies foram: Poaceae (33), Cyperaceae (13), Asteraceae (10), Melastomataceae (7) e Fabaceae (4). Estas famílias são representadas por 67 espécies, constituindo $66,3 \%$ do número total (Tab. 2). Geralmente elas são citadas entre as principais em estudos relacionados com vegetação campestre (Aristeguieta 1968; Pereira et al. 1990; Brandão \& Gavilanes 1994; Brandão et al. 1994; Boldrini et al. 1998). Cyperaceae teve um número de espécies significativamente maior na área preservada, enquanto que Asteraceae teve maior riqueza na área antropizada (Fig. 2). Somente as famílias Poaceae e Cyperaceae totalizaram $45 \%$ das espécies amostradas. Das 33 espécies da família Poaceae, 21 foram encontradas por Filgueiras (1991) em estudo realizado com gramíneas no Distrito Federal. Deste total de espécies comuns, 17 foram encontradas por este autor em área de vereda e as outras quatro foram classificadas como espécies "ruderais" ou "introduzidas na região do cerrado". No presente trabalho elas foram encontradas somente na área antropizada.

Além das famílias com elevado número de espécies, foram amostradas outras como Eriocaulaceae e Xyridaceae, típicas de áreas de veredas (Magalhães 1956), de campos rupestres (Andrade et al. 1986; Brandão et al. 1994), ou de ecossistemas semelhantes às veredas como 
Tabela 2. Espécies em ordem de freqüência relativa da área preservada em uma vereda no município de Uberlândia, MG.

\begin{tabular}{|c|c|c|c|c|c|}
\hline \multirow[b]{2}{*}{ Famílias } & \multirow[b]{2}{*}{ Espécies } & \multicolumn{2}{|c|}{ Área Preservada } & \multicolumn{2}{|c|}{ Área Antropizada } \\
\hline & & $\mathrm{FR}^{*}$ & $\mathrm{CR}^{* *}$ & $\mathrm{FR}^{*}$ & $\mathrm{CR}^{* *}$ \\
\hline POACEAE & Schizachyrium tenerum Nees & 24,272 & 44,818 & 15,947 & 32,891 \\
\hline POACEAE & Echinolaena inflexa Chase & 5,583 & 5,322 & 2,064 & 3,653 \\
\hline POACEAE & Loudetia flammida (Trin.) C.E. Hubbard & 3,641 & 3,651 & 3,752 & 2,589 \\
\hline POACEAE & Erianthus asper Nees & 3,641 & 3,724 & - & - \\
\hline POACEAE & Anthaenantiopsis trachystachya $\mathrm{Mez}$ & 3,398 & 6,153 & 1,876 & 3,862 \\
\hline POACEAE & Andropogon ternatus Nees & 3,398 & 4,874 & 0,750 & 0,736 \\
\hline ASTERACEAE & Ageratum fastigiatum (Gard.) King \& H. Rob. & 3,155 & 2,626 & 4,503 & 4,276 \\
\hline POACEAE & Loudetiopsis chrysothrix (Nees) Conert. & 2,913 & 4,714 & - & - \\
\hline POACEAE & Hypogynium virgatum (Desvaux) Dandy & 2,670 & 1,001 & 5,441 & 4,987 \\
\hline POACEAE & Paspalum cordatum Hackel & 2,427 & 1,473 & 1,313 & 1,639 \\
\hline POACEAE & Hyparrhenia bracteata Stapf & 2,427 & 1,659 & 3,377 & 4,848 \\
\hline POACEAE & Melinis minutiflora Beauv. & 1,699 & 1,349 & - & - \\
\hline LAMIACEAE & Peltodon tomentosus Pohl & 1,699 & 0,734 & 1,126 & 0,237 \\
\hline FABACEAE & Zornia latifolia $\mathrm{Sm}$. & 1,699 & 1,129 & 1,126 & 1,326 \\
\hline POACEAE & Ichnanthus procurrens (Ness ex Trin.) Stieber & 1,699 & 2,631 & - & \\
\hline POACEAE & Arundinella hispida (Willd.) Kuntze & 1,699 & 0,363 & 0,188 & 0,313 \\
\hline POACEAE & Andropogon leptostachyus Benth. & 1,699 & 1,328 & - & - \\
\hline CYPERACEAE & Rhynchospora rugosa (Vahl.) Gale & 1,456 & 0,102 & 0,563 & 0,262 \\
\hline CYPERACEAE & Cyperus odoratus L. & 1,456 & 0,688 & 0,375 & 0,121 \\
\hline LAMIACEAE & Hyptis crenata Pohl ex Benth. & 1,456 & 1,032 & 0,938 & 0,378 \\
\hline POACEAE & Paspalum gardnerianum Nees & 1,214 & 0,308 & 0,563 & 0,368 \\
\hline POACEAE & Panicum cayennense Lam. & 1,214 & 0,407 & 1,876 & 0,419 \\
\hline MELASTOMATACEAE & Tibouchina gracilis (Bonpl.) Cogn. & 1,214 & 0,225 & 3,940 & 1,077 \\
\hline POACEAE & Andropogon selloanus Hack. & 1,214 & 0,366 & 0,938 & 0,837 \\
\hline POACEAE & Panicum sp. & 0,971 & 0,964 & 0,375 & 0,187 \\
\hline CYPERACEAE & Bulbostylis hirtella Nees & 0,971 & 0,187 & 1,126 & 0,777 \\
\hline OCHNACEAE & Sauvagesia racemosa A. St. Hil. & 0,971 & 0,145 & 1,126 & 0,063 \\
\hline MELASTOMATACEAE & Miconia chamissois Naud. & 0,971 & 0,460 & 1,126 & 1,669 \\
\hline CYPERACEAE & Kyllinga odorata H. B. K. & 0,971 & 0,833 & 0,375 & 0,797 \\
\hline POACEAE & Axonopus aureus Beauv. & 0,728 & 0,007 & 0,563 & 0,353 \\
\hline POACEAE & Setaria geniculata (Lam.) Beauv. & 0,728 & 0,230 & 0,750 & 1,134 \\
\hline EUPHORBIACEAE & Croton sclerocalyx (Didr.) M. Arg. & 0,728 & 0,250 & - & - \\
\hline RUBIACEAE & Spermacoce capitata Moc. \& Sesse & 0,728 & 0,436 & 0,188 & 0,086 \\
\hline ARACEAE & Xanthosoma striatipes (Kunth.) Madison & 0,728 & 0,036 & 1,313 & 0,378 \\
\hline EUPHORBIACEAE & Sebastiania myrtilloides (Mart.) Pax & 0,728 & 0,591 & - & - \\
\hline POACEAE & Paspalum hyalinum Nees ex Trin. & 0,485 & 0,349 & 0,563 & 0,338 \\
\hline POACEAE & Andropogon macrothrix Fourn. & 0,485 & 0,160 & 0,375 & 0,137 \\
\hline MELASTOMATACEAE & Miconia albicans (Sw.) Triana & 0,485 & 0,320 & 0,375 & 0,368 \\
\hline LYCOPODIACEAE & Lycopodiella alopecuroides (L.) Cranfill & 0,485 & 0,082 & 1,313 & 1,372 \\
\hline LYTHRACEAE & Cuphea linarioides Cham. \& Schlecht. & 0,485 & 0,005 & - & - \\
\hline XYRIDACEAE & Xyris asperula Mart. & 0,485 & 0,063 & - & - \\
\hline CAESALPINIACEAE & Chamaecrista ramosa Vog. & 0,485 & 0,194 & 0,188 & 0,071 \\
\hline
\end{tabular}


Tabela 2. continuação.

\begin{tabular}{|c|c|c|c|c|c|}
\hline \multirow[b]{2}{*}{ Famílias } & \multirow[b]{2}{*}{ Espécies } & \multicolumn{2}{|c|}{ Área Preservada } & \multicolumn{2}{|c|}{ Área Antropizada } \\
\hline & & $\mathrm{FR}^{*}$ & $\mathrm{CR}^{* *}$ & $\mathrm{FR}^{*}$ & $\mathrm{CR}^{* *}$ \\
\hline POACEAE & $\begin{array}{l}\text { Schizachyrium salzmannii (Trin. ex Steud.) } \\
\text { Nash. }\end{array}$ & 0,485 & 0,141 & 0,938 & 0,348 \\
\hline FABACEAE & Eriosema benthamiana Mart. ex Benth. & 0,485 & 0,145 & - & - \\
\hline ASTERACEAE & Eupatorium laevigatum Lam. & 0,485 & 0,155 & 0,188 & 0,015 \\
\hline MELASTOMATACEAE & Microlicia doryphylla Naud. & 0,485 & 0,068 & - & - \\
\hline IRIDACEAE & Sisyrinchium incurvatum Gard. & 0,485 & 0,165 & 0,563 & 0,323 \\
\hline PASSIFLORACEAE & Passiflora sp. & 0,485 & 0,109 & - & - \\
\hline PIPERACEAE & Piper corintoanum Yunck. \& Derck & 0,485 & 0,756 & 0,563 & 0,353 \\
\hline POACEAE & Schizachyrium condensatum Nees & 0,243 & 0,082 & 0,375 & 0,161 \\
\hline CYPERACEAE & Bulbostylis sp. & 0,243 & 0,039 & 0,375 & 0,055 \\
\hline OCHNACEAE & Sauvagesia linearifolia A. St. Hil. & 0,243 & 0,024 & - & - \\
\hline ACANTHACEAE & Justicia polygaloides (S. Mor.) Lindaw & 0,243 & 0,092 & 0,188 & 0,020 \\
\hline ASTERACEAE & Erechtites hieracifolia (L.) Rafin. & 0,243 & 0,029 & 0,188 & 0,040 \\
\hline ASTERACEAE & Eupatorium burchellii Baker & 0,243 & 0,024 & - & - \\
\hline ONAGRACEAE & Ludwigia nervosa (Poir.) Hara & 0,243 & 0,291 & 0,750 & 0,139 \\
\hline THELYPTERIDACEAE & Thelypteris sp. & 0,243 & 0,048 & - & - \\
\hline ASTERACEAE & Erechtites valeriaeneifolia DC. . & 0,243 & 0,136 & - & - \\
\hline MELASTOMATACEAE & Miconia theaezans Cogn. & 0,243 & 0,412 & - & - \\
\hline XYRIDACEAE & Xyris tenella Kunth & 0,243 & 0,002 & 0,188 & 0,025 \\
\hline POACEAE & Andropogon virgatus Desv. & 0,243 & 0,097 & 3,002 & 1,800 \\
\hline FABACEAE & Desmodium sp. & 0,243 & 0,029 & 0,563 & 0,353 \\
\hline STERCULIACEAE & Byttneria oblongata Pohl & 0,243 & 0,015 & - & - \\
\hline ARECACEAE & Mauritia flexuosa L. f. & 0,243 & 0,291 & 0,563 & 0,434 \\
\hline ASTERACEAE & Vernonia echifolia Mart. ex DC. & 0,243 & 0,073 & - & - \\
\hline POACEAE & Andropogon bicornis Benth. & 0,243 & 0,068 & 0,563 & 0,711 \\
\hline ASTERACEAE & Eupatorium amygdalinum Lam. & 0,243 & 0,073 & - & - \\
\hline BALANOPHORACEAE & Langsdorffia hypogaea Mart. & 0,243 & 0,141 & - & - \\
\hline ASTERACEAE & Vernonia polyanthes Less. & 0,243 & 0,160 & - & - \\
\hline CYPERACEAE & $\begin{array}{l}\text { Rhynchospora globosa (Kunth.) } \\
\text { Roem. \& Schult. }\end{array}$ & 0,243 & 0,019 & 1,126 & 0,464 \\
\hline MELASTOMATACEAE & Trembleya phlogiformis Mart. \& Schr. ex. DC. & 0,243 & 0,102 & 0,188 & 0,161 \\
\hline CYPERACEAE & $\begin{array}{l}\text { Ascolepis brasiliensis (Kunth.)Benth.ex } \\
\text { C.B.Clark. }\end{array}$ & - & - & 4,128 & 4,881 \\
\hline POACEAE & Eriochrysis cayanensis Beauv. & - & - & 3,752 & 1,777 \\
\hline CYPERACEAE & Scleria mitis Berg. & - & - & 1,313 & 0,464 \\
\hline POACEAE & Paspalum notatum $\mathrm{Fl}$. & - & - & 0,938 & 1,135 \\
\hline CYPERACEAE & Rhynchospora velutina (Hunth.) Boeckeler & - & - & 0,938 & 0,877 \\
\hline POACEAE & Poaceae indet. 1 & - & - & 0,750 & 0,393 \\
\hline CYPERACEAE & Eleocharis filiculmis Kunth & - & - & 0,750 & 0,247 \\
\hline XYRIDACEAE & Xyris jupicai Rich. & - & - & 0,750 & 0,237 \\
\hline POACEAE & Eriochrysis laxa Swallen & - & - & 0,750 & 0,292 \\
\hline GLEICHENIACEAE & Dicranopteris flexuosa (Schrad.) Underw & - & - & 0,563 & 1,150 \\
\hline
\end{tabular}


Tabela 2. continuação.

\begin{tabular}{|c|c|c|c|c|c|}
\hline \multirow[b]{2}{*}{ Famílias } & \multirow[b]{2}{*}{ Espécies } & \multicolumn{2}{|c|}{ Área Preservada } & \multicolumn{2}{|c|}{ Área Antropizada } \\
\hline & & $\mathrm{FR}^{*}$ & $\mathrm{CR}^{* *}$ & $\mathrm{FR}^{*}$ & $\mathrm{CR}^{* *}$ \\
\hline ERIOCAULACEAE & Syngonanthus caulescens (Poir.) Ruhl. & - & - & 0,563 & 0,197 \\
\hline CYPERACEAE & Rhynchospora robusta (Kunth.) Boeckeler & - & - & 0,563 & 0,106 \\
\hline ASTERACEAE & Senecio pseudopohlii Cabr. & - & - & 0,375 & 0,025 \\
\hline POLYGALACEAE & Polygala paniculata $\mathrm{L}$. & - & - & 0,375 & 0,131 \\
\hline CYPERACEAE & Fuirena incompleta Nees & - & - & 0,375 & 0,121 \\
\hline CYPERACEAE & Cyperus haspan Benth. & - & - & 0,375 & 0,096 \\
\hline ERIOCAULACEAE & Paepalanthus geniculatus Kunth & - & - & 0,375 & 0,040 \\
\hline POACEAE & Brachiaria decumbens Stapf & - & - & 0,375 & 0,303 \\
\hline ERIOCAULACEAE & Syngonanthus nitens (Bong.) Ruhl. & - & - & 0,375 & 0,091 \\
\hline FABACEAE & Stylosanthes guianensis (Aubl.) Sw. & - & - & 0,375 & 0,131 \\
\hline CECROPIACEAE & Cecropia pachystachya Trec. & - & - & 0,188 & 0,003 \\
\hline POLYGALACEAE & Polygala sp. & - & - & 0,188 & 0,066 \\
\hline MELASTOMATACEAE & Acisanthera alsinaefolia Mart. \& Schr. ex DC. & - & - & 0,188 & 0,131 \\
\hline MALVACEAE & Sida rhombifolia $\mathrm{L}$. & - & - & 0,188 & 0,030 \\
\hline POACEAE & Rhynchelytrum repens (Willd.) Hubbard. & - & - & 0,188 & 0,030 \\
\hline POACEAE & Paspalum lineare Trin. & - & - & 0,188 & 0,156 \\
\hline POLYGALACEAE & Polygala longicaulis H. B. K. & - & - & 0,188 & 0,015 \\
\hline EUPHORBIACEAE & Phyllanthus orbiculatus L. C. Rich. & - & - & 0,188 & 0,061 \\
\hline APIACEAE & Eryngium ebracteatum Lam. & - & - & 0,188 & 0,101 \\
\hline ASTERACEAE & Emilia sonchifolia DC. & - & - & 0,188 & 0,161 \\
\hline
\end{tabular}

*Frequência Relativa **Cobertura Relativa

os "morichales" venezuelanos (Aristeguieta 1968). Os gêneros com maior número de espécies foram Andropogon (6 spp) e Paspalum (5 spp) da família Poaceae, e Rynchospora (4 spp) da família Cyperaceae.

Não ocorreu diferença significativa entre área antropizada e natural, quanto à Freqüência Relativa (FR) (t=3,353; $\mathrm{P}=0,001)$. As dez espécies com maior FR na área preservada somaram $56,05 \%$ e na área antropizada $54,62 \%$ do total (Fig. 3). Destas dez espécies, 90\% são Poaceae, demonstrando ser uma importante família nas veredas, como já havia sido discutido por Brandão \& Gavilanes (1994); Silva Júnior \& Felfili (1996). $\mathrm{Na}$ área antropizada, das dez espécies com maior FR sete foram da família Poaceae, além de Ageratum fastigiatum (Asteraceae), Tibouchina gracilis (Melastomataceae) e Ascolepis brasiliensis (Cyperaceae) (Tab. 2).
Dentre as espécies da família Poaceae, Schizachyrium tenerum foi a que apresentou maior FR nos dois ambientes $(24,27 \%$ no ambiente natural e $15,94 \%$ no antropizado). Echinolaena inflexa, Andropogon ternatus e Erianthus asper foram encontradas na zona de borda da vereda, em solos com baixos teores de umidade e lençol freático mais profundo. E. asper não foi encontrado no ambiente antropizado, enquanto $E$. inflexa e $A$. ternatus tiveram baixa FR. A ausência e/ou diminuição da FR destas espécies no ambiente antropizado pode estar relacionada com uma maior palatabilidade das mesmas para os animais. A distribuição de algumas espécies na vereda ocorreu nos diversos microambientes (zona de borda, meio e fundo). De acordo com Goldsmith (1974), esta distribuição correlacionase com a umidade do solo, proporcionando um gradiente ao longo da vertente. 


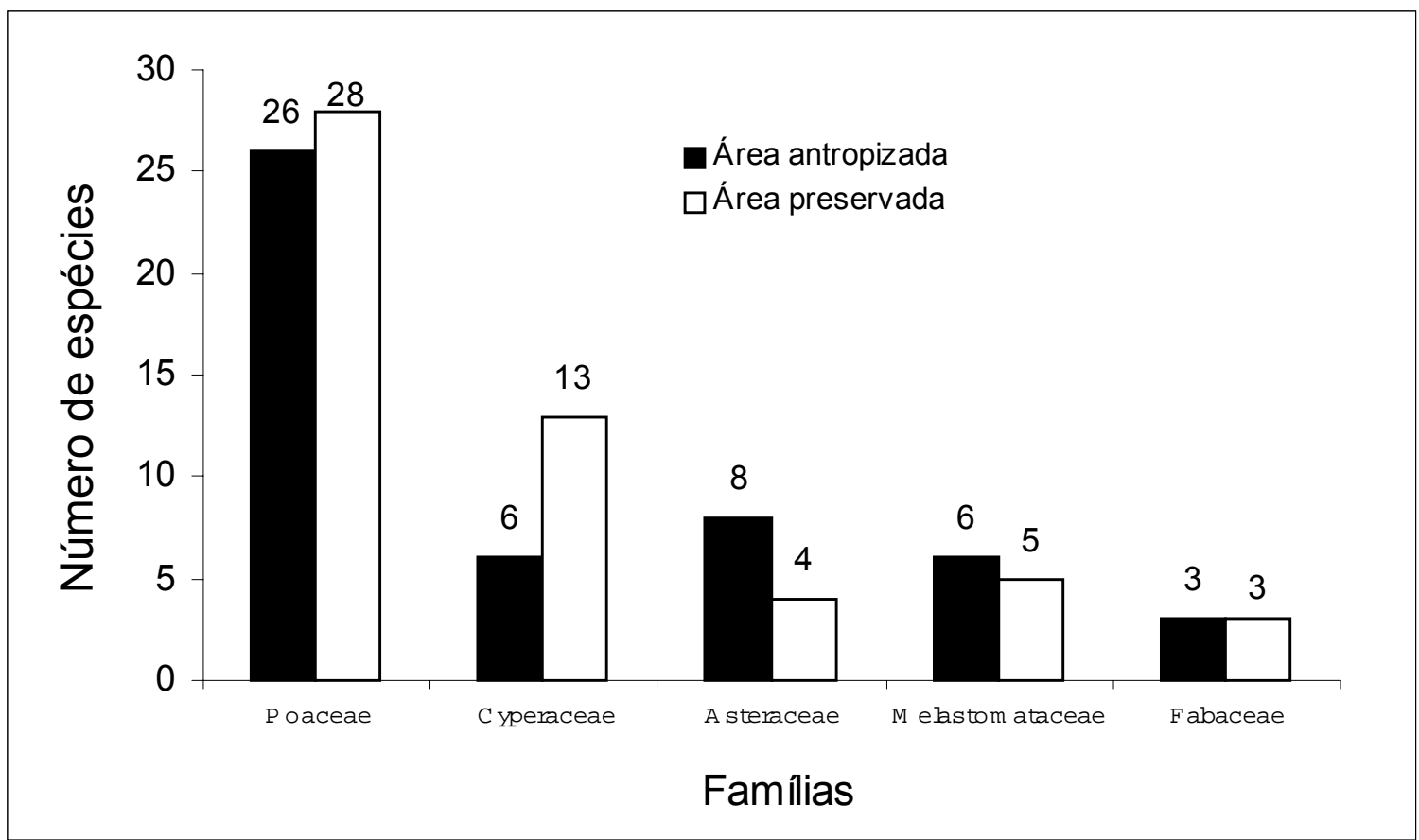

Figura 2. Famílias com maior número de espécies encontradas nas áreas preservada e antropizada em uma vereda no município de Uberlândia, MG.

A ocorrência de Ascolepis brasiliensis (Cyperaceae) e Tibouchina gracilis (Melastomataceae), com altos valores de FR no ambiente antropizado e baixo no preservado, sugere que estas espécies podem estar sendo favorecidas pelo ambiente mais aberto da área em questão, provocado pelo pisoteio do gado e processos erosivos instalados.

As dez espécies com maior Cobertura Relativa (CR) na área preservada somaram 80,3\% do total, enquanto que na área antropizada as mesmas tiveram 72,4\% (Fig. 3). As espécies que apresentaram maior redução de CR na área antropizada foram: Andropogon ternatus e Echinolaena inflexa. Erianthus asper e Ichnanthus procurrens não ocorreram nesse último ambiente.

Ocorreu diferença significativa entre a vertente natural e antropizada da vereda, quanto a cobertura relativa da vegetação $(\mathrm{t}=-0,456$; $\mathrm{P}=0,650$ ). $\mathrm{A}$ menor cobertura vegetal na área antropizada deve-se possivelmente ao efeito do pastejo, ou seja, à perturbação provocada pelo pisoteio e remoção do material verde pelo gado bovino. A maior frequiência de espaços vazios no ambiente antropizado (cerca de 7\%), também aconteceu possivelmente em decorrência dos processos erosivos nas trilhas estabalecidas pelo gado. Segundo Boldrini \& Eggers (1996), o pastejo proporciona o aumento de espécies rizomatosas e estoloníferas em detrimento de espécies cespitosas. Este fato pode ser comprovado pela redução na freqüência e cobertura relativa de algumas espécies na área antropizada, principalmente da família Poaceae, como Echinolaena inflexa, Erianthus asper, Anthaenantiopsis trachystachya e Andropogon ternatus.

A altura média da vegetação nas áreas preservada e antropizada, foi respectivamente 74 $\mathrm{cm}$ e $48 \mathrm{~cm}$, ocorrendo diferença significativa entre estes dois ambientes ( $\mathrm{t}=-4,781 ; \mathrm{P}=0,041)$. Esta diferença ocorreu, possivelmente, devido ao pastejo do gado bovino que remove a biomassa vegetal, principalmente no período relativamente seco. Nesse período, as pastagens, 


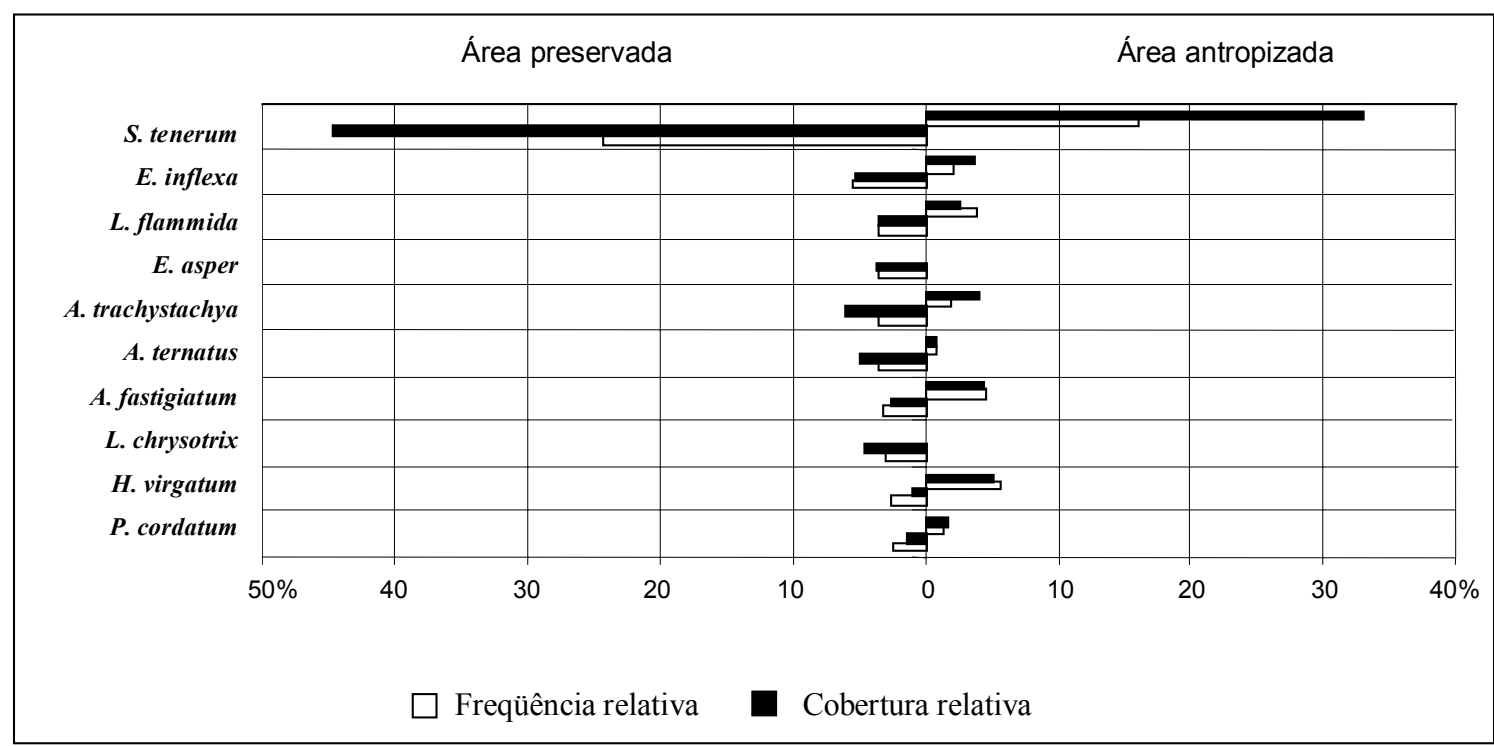

Figura 3. As dez espécies com maior freqüência e cobertura relativa da área preservada e seus respectivos valores na área antropizada em uma vereda no município de Uberlândia, MG.

na periferia da vereda, são escassas e o gado busca as áreas de maior umidade para sua alimentação.

Quanto à porcentagem média de matéria viva da vegetação herbácea, medida nos meses de março, abril, maio e junho, nos três transectos, não foi observada diferença significativa $(\mathrm{t}=0,981 ; \mathrm{P}=0,019)$, entre as duas áreas. No lado antropizado o valor foi de $66,65 \%$ e no preservado de $55,82 \%$. A maior porcentagem de matéria viva no lado antropizado, relaciona-se com o constante rebrotamento vegetal proporcionado pelo pastejo. Na zona de borda da vereda, próximo ao cerrado, a porcentagem de matéria viva foi menor em decorrência dos menores valores de umidade no solo.

Portanto, devido à pequena diferença nos valores de $\mathrm{pH}$ e de bases trocáveis entre as vertentes antropizada e preservada, não foi possível determinar variações, quanto às características químicas, dos solos nos dois ambientes. Porém, as diferenças entre as duas áreas, quanto a alguns parâmetros fitossociológicos, demonstram que a flora da vereda é sensível à antropização. Alterações na riqueza vegetal, devido às espécies invasoras e na freqüência e cobertura pelo pastejo e pisoteio bovino, podem estar colocando em risco a manutenção do regime hídrico, assim como da diversidade vegetal nativa, dessa fitocenose situada em áreas de nascentes.

\section{Referências bibliográficas}

Andrade, P.M., Gontijo, T.A. \& Grandi, T.S.M. 1986. Composição florística e aspectos estruturais de uma área de "Campo Rupestre" do Morro do Chapéu, Nova Lima, Minas Gerais. Revista Brasileira de Botânica 9: 13-21.

Aristeguieta, L. 1968. Consideraciones sobre la flora de los Morichales Llaneros al Norte del Orinoco. Acta Botanica Venezuelana 3: 3 - 22.

Baccaro, C.A.D. 1994. As unidades geomorfológicas e a erosão nos chapadões do município de Uberlândia. Sociedade \& Natureza 6 (11/12): 19-33.

Belsky, A.J. 1992. Effects of grazing, competition, disturbance and fire on species composition and diversity in grassland communities. Journal Vegetation Science 3: 187-200.

Boaventura, R.S. 1988. Preservação das veredas; síntese. In.: Encontro Latino Americano: Relação Ser Humano/Ambiente. Anais. p. 109 - 119.

Boldrini, I.I. \& Eggers, L. 1996. Vegetação campestre do sul do Brasil: Dinâmica de espécies à exclusão do gado. Acta Botanica Brasilica 10 (1): 37-50.

Boldrini, I.I., Miotto, S.T.S., Longhi-Wagner,H.M., 
Pillar,V.P. \& Marzall, K. 1998. Aspectos florísticos e ecológicos da vegetação campestre do Morro da Polícia, Porto Alegre, RS, Brasil. Acta Botanica Brasilica 12 (1): 89-100.

Brower, J.F. \& Zar, J.H. 1984. Field \& laboratory methods for general ecology - Dubuque, Iowa.

Brasil. 1992. Resoluções CONAMA de 1984 a 1991. SEMAM / IBAMA. Brasília, DF. $4^{\mathrm{a}}$ ed. 245 p.

Brandão, M. \& Gavilanes, M.L. 1994. Cobertura Vegetal da Microrregião 178 (Uberaba), Minas Gerais, Brasil. Daphne 4 (2): 29-57.

Brandão, M., Gavilanes, M.L. \& Araújo, M.G. 1994. Aspectos físicos e botânicos de campos rupestres do Estado de Minas Gerais-I. Daphne 4 (1): 17-38.

Carvalho, P.G.S. 1991. As veredas e sua importância no Domínio dos Cerrados. Informe Agropecuário 168: $47-54$

Embrapa. 1979. Serviço nacional de levantamento e conservação dos solos. Manual de métodos de análise do solo. Rio de Janeiro, RJ.

Embrapa. 1982. Serviço nacional de levantamento e conservação dos solos. Epamig (Belo Horizonte, MG). Levantamento de reconhecimento de média intensidade dos solos e avaliação da aptidão agrícola das terras do Triângulo Mineiro. Boletim de pesquisa 1. Rio de Janeiro, RJ. p.34-43.

Embrapa. 1999. Centro Nacional de Pesquisa de Solos (Rio de Janeiro, RJ). Sistema brasileiro de classificação de solos. - Brasília : Embrapa Produção de Informação; Rio de Janeiro : Embrapa Solos. xxvi, 412p. : il.

Ferreira, M.B. 1980. O cerrado em Minas Gerais, gradações e composição florística. Informe Agropecuário 61: 4-8.

Filgueiras, T.S. 1991. A floristic analysis of the Gramineae of Brazil's Distrito Federal and list of the species occurring in the area. Edinburgh Journal Botany 48: 73-80.

Fonseca, V.S. \& Silva, I.M. 1998. Etnobotânica: base para conservação. Workshop Brasileiro de Etnobotânica, 136p.

Gavilanes,M.L. \& D’angieri Filho,C.N. 1991. Flórula ruderal da cidade de Lavras, MG. Acta Botanica Brasilica 5 (2): 77-88.

Goldsmith, F.B. 1974. Multivariate analysis of tropical grassland communities in Mato Grosso, Brazil. Journal of Biogeography 1: 111-122.

Lima, S.C. 1996. As veredas do Ribeirão do Panga no Triângulo Mineiro e a evolução da paisagem. Tese de Doutorado. USP, São Paulo.

Lima, S.C. \& Queiroz Neto, J.P. 1996. As veredas e a evolu- ção do relevo. Sociedade \& Natureza 15: 481- 488.

Lima, S.C., Rosa, R. \& Feltran Filho, A. 1989. Mapeamento do uso do solo no Município de Uberlândia-MG, através de imagens TM / LANDSAT. Sociedade \& Natureza 1: 127 - 145.

Magalhães, G.M. 1956. Características de alguns tipos florísticos de Minas Gerais. Revista Brasileira de Biologia 1: 76-92.

Melo, D.R. 1992. As veredas nos planaltos do noroeste mineiro; caracterizações pedológicas e os aspectos morfológicos e evolutivos. Dissertação de Mestrado. UNESP, Rio Claro.

Mendonça, R.C., Felfili, J.M., Walter, B.M.T., Silva Júnior, M.C., Rezende, A.V., Filgueiras, T.S. \& Nogueira, P.E. 1998. Flora Vascular do Cerrado. In: Cerrado ambiente e flora. EMBRAPA-CPAC. 556p.

Milchunas, D.G., Sala, O.E. \& Lauenroth, W.K. 1988. A generalized model of the effects of grazing by large herbivores on grassland comunity structure. American Naturalist 132: 87-106.

Minas Gerais.1988. Lei no 9.682 de 12 de outubro de 1988 Declara de interesse comum e de preservação permanente os ecossistemas das veredas no Estado de Minas Gerais. Belo Horizonte, MG. V. 193.

Mueller-Dombois, D. \& Ellenberg, H. 1974. Aims and methods of vegetation ecology. New York, Willey $\&$ Sons.

Pandey, C.B. \& Singh, J.S. 1991. Influence of grazing and soil conditions on secondary savanna vegetation in India. Journal Vegetation Science 2: 95-102.

Pedrotti, D.E. \& Guarim Neto, G. 1998. Flora Ruderal da Cidade de Cuiabá, Mato Grosso, Brasil. Acta Botanica Brasilica 12 (2): 135-143.

Pereira, B.A.S., Mendonça, R.C., Filgueiras, T.S., Paula, J.E. \& Heringer, E.P. 1990. Levantamento florístico da Área de Proteção Ambiental (APA) da bacia do Rio São Bartolomeu, DF. Anais do XXXVI Congresso Brasileiro de Botânica 1: 419-492.

Ramirez, N. \& Brito, Y. 1990. Reproductive of a tropical palm swamp community in the Venezuelan llanos. American Journal of Botany 77: 1260 - 1271.

Ramos, M.V.V. 2000. Veredas do Triângulo Mineiro: solos, água e uso. Dissertação de Mestrado. UFLA, Lavras.

Ricklefs, R.E. 1996. A Economia da Natureza. $3^{\text {a }} \mathrm{ed}$. Ed. Guanabara Koogan S.A. Rio de Janeiro. 470 p.

Rosa, R., Lima, S.C. \& Assunção, W.L. 1991. Abordagem preliminar das condições climáticas de Uberlândia, MG. Sociedade \& Natureza 3(5 e 6): 91-108.

Schiavini, I. \& Araújo, G.M. 1989. Considerações so- 
bre a vegetação da Reserva Ecológica do Panga (Uberlândia). Sociedade \& Natureza 1: 61-66. Silva Júnior, M.C. \& Felfili, J.M. 1996. A vegetação da Estação Ecológica de Águas Emendadas. Ins- tituto de Ecologia e Meio Ambiente do Distrito Federal. Brasília, DF. 43 p.

Zar, J.H. 1984. Biostatistical Analysis. $2^{\text {a }}$ ed. PrenticeHall, Inc., New Jersey. 718 p. 